\title{
Improving the Efficiency of Localization-Oriented Network Adjustment in Wireless Sensor Networks
}

\author{
Tao Chen, Deke Guo, Zheng Yang, Honghui Chen, and Xueshan Luo
}

\begin{abstract}
In real-world deployment, some wireless sensor networks are not entirely localizable. A remedy for such situation is to adjust the network to make it well-prepared for localization. Previous studies mainly focus on adding measurable edges into the network, so as to enhance localizability, but they introduce redundant distance measurement. This letter presents a new theoretic finding in 2-connected graphs, and proposes accompanying network adjustment approach, which can significantly reduce the number of added edges from $(d-1)^{2} n$ to only $2 n$. Simulation results show that our approach outperforms previous work in adjustment efficiency.
\end{abstract}

Index Terms-Localization, wireless sensor networks, adjustment, network deployment.

\section{INTRODUCTION}

$\mathbf{L}$ OCALIZATION is an active and important research problem in wireless sensor networks. Due to resource limitation of common off-the-shelf sensor nodes, a lightweighted and inexpensive localization algorithm is essential to determine the location of each sensor node. Such requirement leads to the research of in-network localization [1], [2], in which some special nodes (called beacons or anchors) know their global locations and the rest determine their coordinates by measuring the distances to their neighbors. These algorithms perform well in dense networks, however, they cannot determine the locations of all nodes in sparse networks. Theoretic findings have shown that, a network can be partially localizable due to hardware or deployment constraints[3], [4]. Such networks should be adjusted before the process of localization.

For many sensor networks (such as those deployed in unattended area), it is important to adjust the network configuration without extra devices. A convenient way is to increase the distance ranging capability of sensor nodes. Enhanced nodes can measure the distances to a larger number of nearby nodes, thus bringing in more distance constraints and enhancing localizability. Such enhancement is feasible in many rangebased localization approaches using Time of Arrival (ToA) and Received Signal Strength (RSS), since it can be achieved by augmenting the transmitter power output. Based on the

Manuscript received June 23, 2011. The associate editor coordinating the review of this letter and approving it for publication was F. Granelli.

T. Chen, D. Guo, H. Chen, and X. Luo are with the Key Lab of Information System Engineering, College of Information Systems and Management, National University of Defense Technology, China (e-mail: \{emilchenn, guodeke, chh0808\}@gmail.com,xsluo@nudt.edu.cn).

Z. Yang is with the Hong Kong University of Science and Technology, Hong Kong, and TNLIST, School of Software, Tsinghua University, China (e-mail: yangzh@cse.ust.hk).

This work is supported in part by the NSF China under Grant No. 60903206, and the Preliminary Research Foundation of the National University of Defense Technology.

Digital Object Identifier 10.1109/LCOMM.2011.081211.111345 above discussion, Chen et al. [5] propose a "localizabilityaided localization approach"(denoted by LAL) for network adjustment. The upper bound on the number of additional edges in LAL can be approximated by $(d-1)^{2} n$, where $d$ is the average degree and $n$ is the number of non-localizable nodes. However, LAL is not optimal, and some unnecessary edges are added into the network. Those edges are redundant, cost considerable time and energy for distance measurement, thus reduce the efficiency of network adjustment. Motivated by this, we examine the theoretical results of localization and localizability, and find a new property of 2-connected graphes. Based on our finding, we propose a network adjustment approach, which can significantly reduce the number of additional edges. The number of added edges is bounded by $2 n$. The result is a significant progress to reduce redundant edges which are not necessary to be measured. And this may effectively improve the efficiency of localization-oriented network adjustment.

\section{Problem Statements}

This letter focuses on the problem of how to adjust a wireless sensor network to make it localizable. The ground truth of a wireless sensor network can be modeled by a distance graph $G=(V, E)$, where $V$ denotes the set of vertices (e.g. sensor nodes) and $E$ denotes the set of edges. For $i, j \in V,(i, j) \in E$ if the distance between $i$ and $j$ can be measured or both of them are in known locations (e.g. beacons). We assume $G$ is connected and has at least four vertices, which is deployed in 2D space. A network (or a graph) is generic if the node (or vertex) coordinates are algebraically independent. Like many previous work [2], [3], [4], [6], [5], we focus on generic cases in our study.

Without extra device, the aim of localization-oriented network adjustment is to add edges into the corresponding distance graph $G$ and ensure that the new graph is entirely localizable. We prefer the solution with less extra edges, because redundant edges cause meaningless adjustment and accompanying cost for distance measurement.

\section{TheORETIC FOUNDATIONS}

A graph is called rigid if one cannot continuously deform its realizations while preserving distance constraints, and redundantly rigid if it remains rigid upon removal of any single edge [7]. Based on the graph rigidity theory, network localizability [3] and node localizability [4] are studied, which solve the location-uniqueness of a network or a single node, respectively.

Lemma 1 ([8]). A graph with $n \geq 4$ vertices is globally rigid in 2 dimensions if and only if it is 3-connected and redundantly rigid. 
Theorem 1 ([3]). A network is uniquely localizable if and only if its distance graph is globally rigid and it contains at least three beacons.

Theorem 2 ([4]). In a distance graph $G=(V, E)$ with a beacon set $B \subset V$ where $|B| \geq 3$, a vertex is localizable if it is included in the redundantly rigid component inside which there are three vertex-disjoint paths to three vertices in $B$.

Based on Theorem 1, Anderson et al. present the following result.

Theorem 3 ([6]). Let $G=(V, E)$ be a 2-edge-connected graph in 2-dimension space. $G^{2}$ is obtained from $G$ by adding edges between two-hop vertex pairs. Then $G^{2}$ is globally rigid.

An localizability-aided approach (LAL) [5] has been proposed recently, which based on the following theorem.

Theorem 4 ([5]). Suppose $G=(V, E)$ is a 2-connected graph with a beacon set $B$, where $B \subset V$ and $|B| \geq 3$. Let $V_{N}$ denote the set of non-localizable vertices, and $E_{N}$ denote the set of edges $(i, j), i \in V_{N}$ and $(i, j) \in E^{2}$. Then, $G^{\prime}=$ $\left(V, E \cup E_{N}\right)$ is localizable.

LAL decomposes the distance graph of a network into 2connected components, and adds new edges mainly based on Theorem 3 and Theorem 4 . The two theorems provide sufficient conditions to make the graph localizable, however, they also introduce unnecessary edges into the graph, especially in non-trivial 2-connected subgraph. In the worst case, Theorem 4 adds $(d-1)^{2} n$ edges, where $d$ is the average degree of the graph, and $n$ is the number of non-localizable vertices.

To reduce the number of added edges in network adjustment, we try to find new properties of localizable graph, and propose the following theorem.

Theorem 5. Suppose $P=(V, E)$ is a path ${ }^{1}$, which starts from a localizable vertex to another localizable one and has at least three localizable vertices. Let $V_{N}$ denote the set of nonlocalizable vertices, and $E_{N}$ denote the set of edges $(i, j), i \in$ $V_{N}$ and $(i, j) \in E^{2}$. Then, $P^{\prime}=\left(V, E \cup E_{N}\right)$ is localizable.

Proof: Assume $|V|>3$, since the result is trivial if $|V|=3$. Suppose $P$ has vertices $v_{1}, v_{2}, \ldots, v_{k}$, and $v_{1}$ and $v_{k}$ are two ends of $P$. Consider the worst case that $P$ has only three localizable vertices, thus $P^{\prime}$ has edge $\left(v_{1}, v_{2}\right),\left(v_{1}, v_{3}\right),\left(v_{2}, v_{3}\right),\left(v_{2}, v_{4}\right), \ldots,\left(v_{k-2}, v_{k}\right),\left(v_{k-1}, v_{k}\right)$. Let $G$ be the distance graph of $P^{\prime}$. $G$ has at least one more edge $\left(v_{1}, v_{k}\right)$. Since $G$ has three localizable vertices, we shall show that $G$ is 3 -connected and redundantly rigid. As noted in Lemma 1 and Theorem 1, these properties are necessary and sufficient to establish localizability.

Consider the existence of paths in $G$ between $v_{i}$ and $v_{i+2 m}$ for any $i$ and $m$ with $i+2 m \leq k$. Three paths which have no common vertices other than end vertices are: $v_{i} v_{i+1} v_{i+3} \ldots v_{i+2 m-1} v_{i+2 m}, v_{i} v_{i+2} v_{i+4} \ldots v_{i+2 m}$ and $v_{i} v_{i-1} \ldots v_{1} v_{k} v_{k-1} \ldots v_{i+2 m}$. Likewise if we consider paths between $v_{i}$ and $v_{i+2 m+1}$, we can find three similar paths. This establishes the 3-connectivity of $G$.

\footnotetext{
${ }^{1}$ A path without repeated vertices is a simple path. We omit the word simple in this letter for convenience, since all paths hereafter are simple paths.
}

Suppose an edge $e$ is removed from $G$. If $e=$ $\left(v_{1}, v_{k}\right)$, the remaining sequence of triangles in $G-e$ are: $v_{1} v_{2} v_{3}, v_{2} v_{3} v_{4}, \ldots v_{k-2} v_{k-1} v_{k}$. $G-e$ is spanned by triangles, each of which shares two vertices with its former one. So we have $G-e$ is rigid. In other cases, if $\left(v_{j}, v_{j+1}\right)$ or $\left(v_{j}, v_{j+2}\right)$ (for any $j$ ) is removed, it is easy to find that all subgraphs in $G-e$ has no more than $2 n^{\prime}-3$ edges, where $n^{\prime}$ is the number of vertices in the subgraph. Since $G-e$ has at least $2 k-3$ edges, $G-e$ is rigid according to the result (Corollary 2.3) in [9] (we omit the detail here for space limitation). Hence the redundant rigidity of $G$ is established.

If $P$ has more than 3 localizable vertices, it can be decoupled into multiple sub-paths of the worst case and the aforementioned result also holds.

Theorem 5 hints that, if we can find an above mentioned path for each non-localizable vertex, we may bound the number of extra edges to $2 n$, where $n$ is the number of nonlocalizable vertices.

\section{Path-Based Adjustment}

In this section, we focus on how to use our theoretic finding to adjust a 2-connected graph. We assume the localizability information of each vertex is available (by using Theorem 2 ), and there should exist at least three beacons in a given 2-connected graph $G$, otherwise $G$ cannot be adjusted to be entirely localizable. We also assume at least two beacons are adjacent, since it can be easily achieved in deployment. Let $v_{1}, v_{2}$ and $v_{3}$ denote three beacons, and $v_{2}$ is adjacent to $v_{3}$. Based on Theorem 5, we propose a path based adjustment algorithm as shown in Algorithm 1.

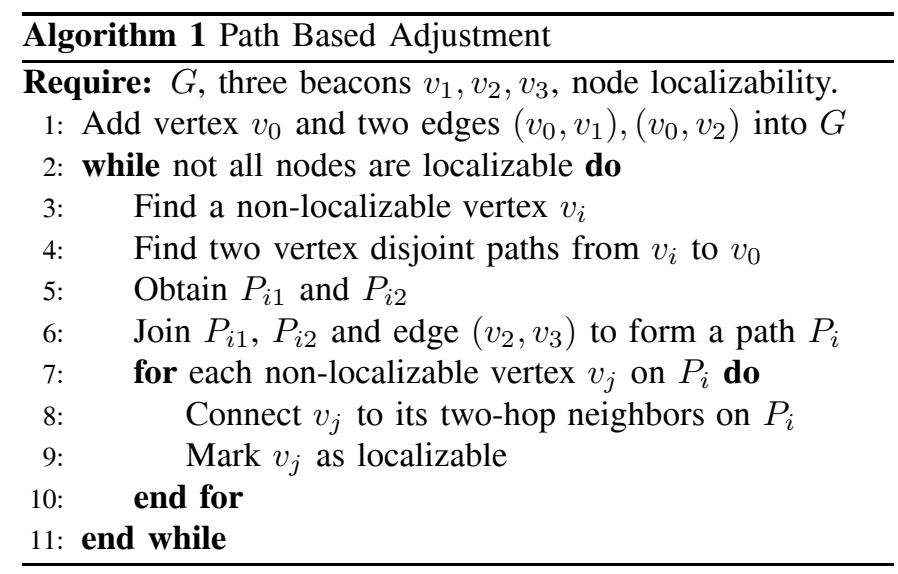

At first, a virtual vertex $v_{0}$ and two edges $\left(v_{0}, v_{1}\right)$ and $\left(v_{0}, v_{2}\right)$ are added into $G$, resulting a new graph denoted by $G_{0}$. There is a path $P_{12}$ in $G$ which connects $v_{1}$ and $v_{2}$. As shown in Fig. 1, such a path and two edges $\left(v_{0}, v_{1}\right)$ and $\left(v_{0}, v_{2}\right)$ can join together and form a cycle in $G_{0}$. Since $G$ is 2-connected and the cycle has no less than two vertices in $G, G_{0}$ is also 2 -connected. For any non-localizable vertex $v_{i}$ in $G$, we can find two vertex-disjoint paths from $v_{i}$ to $v_{0}$ in $G_{0}$ by applying popular network flow algorithms in line 4. Because there are only two edges incident to $v_{0}$, the two vertex-disjoint paths must pass $v_{1}$ and $v_{2}$, respectively. So it is easy to find two paths $P_{i 1}$ and $P_{i 2}$, which have no common vertex and connect $v_{i}$ to $v_{1}$ and $v_{2}$, respectively. We can join 


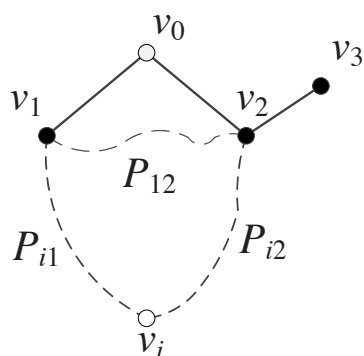

Fig. 1. An example of finding paths for adjustment. $v_{1}, v_{2}$ and $v_{3}$ are localizable vertices. $v_{0}$ is the virtual vertex. $v_{i}$ is a non-localizable vertex.

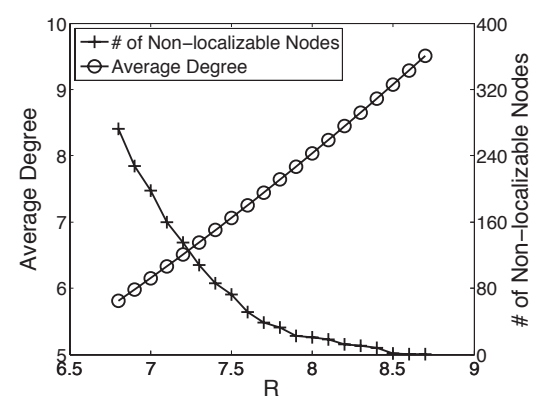

Fig. 2. Average degree and the number of nonlocalizable nodes in different network settings.

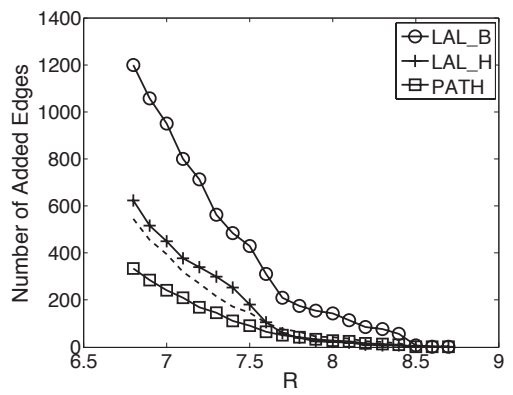

Fig. 3. Average number of added edges by using LAL and PATH. Dashed line denotes twice the average number of non-localizable nodes in the original topology.
$P_{i 1}, P_{i 2}$ and $\left(v_{2}, v_{3}\right)$ to get a path in $G$ denoted by $P_{i}$, which starts from $v_{1}$ and ends at $v_{3}$. In trivial cases, $v_{3}$ is in $P_{i 1}$ or $P_{i 2}$, we only need to join $P_{i 1}$ and $P_{i 2}$ together. According to Theorem 5, adjustment can be made based on $P_{i}$ to make all non-localizable nodes on the path (including $v_{i}$ ) localizable. Since $v_{i}$ is arbitrarily chosen, all non-localizable vertices can be made localizable in the same way.

\section{Simulation Results}

We compare the proposed adjustment solution, denoted as PATH, with two implementations of LAL. LAL_B denotes the LAL with basic approach, while LAL_H denotes the one with heuristic algorithm. The simulations are conducted based on TOSSIM $^{2}$ and Matlab 7.6. We use the tools in TOSSIM to generate positions for 400 nodes, which are uniformly distributed in a square $[0,100]^{2}$. Given the topologies, we use Matlab in our simulations for ease of implementation. The disk model with a radius is adopted for distance measurement. We integrate results from 100 network instances, with three beacons in each instance. The original distance measurement range is denoted by $R$. For each instance, we vary the value of $R$ from 6.8 to 8.7 stage-by-stage with a step length of 0.1 .

Fig. 2 plots the average node degree and the number of non-localizable nodes in the original topology in different settings. The minimal value of $R$ is 6.8 , since it is difficult to find random topologies which can fulfill the connectivity requirements with a smaller distance measurement range. When $R$ is set a small value, the network has a small average degree, and most nodes are non-localizable. As $R$ increases, the average node degree increase linearly, and the number of non-localizable nodes decreases. Almost all network instances are localizable when $R=8.5$. Fig. 3 plots the average number of added edges, resulting from three adjustment approaches. All three approaches add fewer edges as $R$ increases, since more nodes are localizable. Our solution, PATH, can effectively reduce the number of extra edges, especially in sparse topologies, thus outperforms other two previous approaches. The fundamental reason behind this result is that, LAL_B requires that each non-localizable node connects to all its two-hop neighbors, while PATH only needs to add two edges for a non-localizable node. Although the heuristic scheme used in LAL_H can reduce some redundant edges, it still cannot approach PATH, especially in sparse topologies. PATH also shows high efficiency in dense topology. It still needs fewer extra edges than LAL_B, and has almost the same performance as LAL_H. The performance difference is not so large as in sparse topology, since only a few nodes are non-localizable. We can also find that the number of added edges of PATH is always fewer than twice the number of non-localizable nodes. The reason is that two non-localizable nodes may share an extra edge in the adjustment process. The results are in accordance with the analysis in Section III.

\section{CONCLUSION}

We present theoretical result and accompanying algorithm to improve the efficiency of localization-oriented network adjustment. Our approach can reduce the number of additional edges from $(d-1)^{2} n$ to $2 n$, where $n$ is the number of nonlocalizable nodes. Simulation results show that our approach outperforms the previous work. For future work, we intend to implement our approach in a distributed manner.

\section{REFERENCES}

[1] N. B. Priyantha, A. Chakraborty, and H. Balakrishnan, "The Cricket location-support system," in Proc. MobiCom 2000, pp. 32-43.

[2] D. K. Goldenberg, P. Bihler, M. Cao, J. Fang, B. D. O. Anderson, A. S. Morse, and Y. R. Yang, "Localization in sparse networks using sweeps," in Proc. MobiCom 2006, pp. 110-121.

[3] T. Eren, D. K. Goldenberg, W. Whiteley, Y. R. Yang, A. S. Morse, B. D. O. Anderson, and P. N. Belhumeur, "Rigidity, computation, and randomization in network localization," in Proc. INFOCOM 2004.

[4] Z. Yang and Y. Liu, "Understanding node localizability of wireless ad-hoc networks," in Proc. INFOCOM 2010.

[5] T. Chen, Z. Yang, Y. Liu, D. Guo, and X. Luo, "Localization in nonlocalizable sensor and ad-hoc networks: a localizability-aided approach," in Proc. INFOCOM 2011.

[6] B. D. Anderson, P. N. Belhumeur, T. Eren, D. K. Goldenberg, A. S. Morse, W. Whiteley, and Y. R. Yang, "Graphical properties of easily localizable sensor networks," Wireless Netw., vol. 15, no. 2, pp. 177-191, 2009.

[7] G. Laman, "On graphs and rigidity of plane skeletal structures," $J$. Engineer. Math., vol. 4, pp. 231-340, 1970.

[8] B. Jackson and T. Jordan, "Connected rigidity matroids and unique realizations of graphs," J. Comb. Theory Ser. B, vol. 94, no. 1, pp. 1$29,2005$.

[9] D. J. Jacobs and B. Hendrickson, "An algorithm for two-dimensional rigidity percolation: the pebble game," J. Computational Physics, vol. 137, pp. 346-365, 1997.

${ }^{2} \mathrm{http}: / /$ www.cs.berkeley.edu/ pal/research/tossim.html 